\title{
Notch Signaling: A Novel Therapeutic Target for Cancer
}

\section{Yuefei Yu*}

Division of Hematology and Oncology, Department of Internal Medicine, Texas Tech University Health Sciences Center, Lubbock, Texas, USA

Notch signaling pathway is highly conserved in evolution in the signal transduction pathway, in which it regulates stem-cell maintenance, cell proliferation, differentiation and apoptosis in almost all the tissues and organs. Notch signaling plays a critical role in cell fate decision in many developmental systems. Notch-mediated signaling can expand and cure molecular differences between adjacent cells, and ultimately determine the fate of cells. Notch receptors are type I transmembrane heterodimeric receptors that widely exist in all known animal cells. There are four Notch receptors (Notch1-4), three Delta-like ligands (Dll1, Dll3, and Dll4), and two ligands of the Jagged family (Jag1 and Jag2) in mammals [1].

Aberrant Notch signaling has been implicated in the uncontrolled growth of malignant cells, which might function as an oncogene. The further understanding of Notch signaling in normal development and malignant transformation may open up a new avenue for developing novel cancer therapeutics. The first evidence for the involvement of Notch signaling in cancer came from T-cell acute lymphoblastic leukaemia (T-ALL). The progress in the characterization of the mechanisms that mediate the oncogenic activity of Notch signaling make it possible to develop new anti-Notch therapies in the treatment of T-ALL. The high prevailing of Notch1 mutations in T-ALL makes it an ideal target for small molecules therapy. In the gross, Notch signaling could be inhibited at several levels: ligand binding, endocytosis, proteolysis or transcriptional activity [2]. A multi-subunit protease, $\gamma$-secretase, is critical in the processing of Notch. $\gamma$-secretase inhibitors (GSIs), the small molecules, are considered to be the most promising therapeutic agents, which could block the growth of T-ALL cells. Now GSIs are widely used to inhibit the Notch signaling in basic research and clinical trials. It has been reported that the combination of GSI with intravenous chemotherapy had a good antitumor activity in several tumor types including pancreas and nasopharyngeal carcinoma. The study showed that Notch protein expression was lower in patients who achieved prolonged stable disease or partial response [3]. GSI is also a promising therapeutic avenue in brain tumors, specifically Glioblastoma multiforme (GBM). Wu et al. [4] reported that phosphorylation state of AKT and STAT3 was reduced by arsenic trioxide (ATO) largely, and both p-AKT and p-STAT3 levels were totally blocked by ATO plus GSI treatment. ATO targeted Notch signaling pathway and induced apoptosis through inhibition of phosphorylation of AKT and STAT3. These results suggested that ATO could inhibit the phosphorylation and activation of AKT and STAT3 through Notch signaling blockade.

On the other hand, the development of pharmacological inhibitors that specifically target other components of the Notch pathway would permit more-flexible and specific therapeutic strategies. Zhu et al. [5] showed that elevated expression of Jagged-1 and Notch- 1 in neoplastic epithelial cells is associated with a biologically aggressive, metastatic phenotype in prostate cancer cells. The findings suggest that Jagged-1 and Notch-1 may serve as useful markers in distinguishing indolent and aggressive prostate cancer and Jagged1-Notch1 signaling could be a potential therapeutic target for the treatment of prostate cancer. Zhang et al. [6] identified that the Notch/Dll4 pathway activated by
VEGF initiated a novel signaling cross-talk between acute myeloid leukemia (AML) and endothelial cells. The Notch/Dll4 pathway enhances the activity of angiogenesis in AML. The elucidation of the molecular mechanisms might facilitate the development of novel therapeutic strategies for AML. Grieselhuber et al. [7] have reported that the expression of JAG1 and activation of Notch signaling are common events in the development of both murine and human acute promyelocytic leukemia (APL), which demonstrated a role for Notch signaling in the pathogenesis of APL. Moreover, both genetic and pharmacologic interference of Notch signaling blocked the enhanced self-renewal seen in hematopoietic stem/progenitor cells. Interestingly, the blockage of Notch signaling could inhibit the growth of murine APL tumors in vitro and in vivo. These findings expand the role of Notch signaling in hematopoietic diseases.

In another recent study, it was described that high level of Notch3 expression was significantly correlated with non-small-cell lung cancer (NSCLC) progression and unfavorable prognosis. The nuclear Notch3 expression is an independent prognostic biomarker of overall survival for NSCLC, which may be used as a clinical parameter for prediction prognostication of NSCLC patients. Therefore, targeting the Notch3 pathway may be used for therapeutic strategies for NSCLC instead of conventional chemotherapy or radiation therapy [8]. Furthermore, the recent reports have described the involvement of aberrant Notch signaling in the pathogenesis of solid tumors and tumor angiogenesis, implying a new role for anti-Notch signaling in human cancer therapeutics.

\section{References}

1. Lobry C, Oh P, Aifantis I (2011) Oncogenic and tumor suppressor functions of Notch in cancer: it's NOTCH what you think. J Exp Med 208: 1931-1935

2. Grabher C, von Boehmer H, Look AT (2006) Notch 1 activation in the molecular pathogenesis of T-cell acute lymphoblastic leukaemia. Cancer 6: 347-359

3. Richter S, Bedard PL, Chen EX, Clarke BA, Tran B, et al. (2013) A phase I study of the oral gamma secretase inhibitor R04929097 in combination with gemcitabine in patients with advanced solid tumors (PHL-078/CTEP 8575). Invest New Drugs.

4. Wu J, Ji ZY, Liu HL, Liu YH, Han DY, et al. (2013) Arsenic trioxide depletes cancer stem-like cells and inhibits repopulation of neurosphere derived from glioblastoma by downregulation of Notch pathway. Toxicol Lett 220: 61-69

5. Zhu H, Zhou XC, Redfield S, Lewin J, Miele L (2013) Elevated Jagged-1 and Notch-1 expression in high grade and metastatic prostate cancers. Am J Transl Res 5: 368-378

6. Zhang JR, Ye JJ, Ma D, Liu N, Wu H, et al. (2013) Cross-talk between leukemic

*Corresponding author: Yuefei Yu, Division of Hematology and Oncology, Department of Internal Medicine, Texas Tech University Health Sciences Center, Lubbock, Texas, USA, Tel: 806-559-9076; E-mail: yuefei.yu@ttuhsc.edu

Received May 24, 2013; Accepted May 27, 2013; Published May 30, 2013

Citation: Yu Y (2013) Notch Signaling: A Novel Therapeutic Target for Cancer. Single Cell Biol 2: e120. doi:10.4172/2168-9431.1000e120

Copyright: (c) $2013 \mathrm{Yu}$ Y. This is an open-access article distributed under the terms of the Creative Commons Attribution License, which permits unrestricted use, distribution, and reproduction in any medium, provided the original author and source are credited. 
and endothelial cells promotes angiogenesis by VEGF activation of the Notch/ DII4 pathway. Carcinogenesis 34: 667-677

7. Grieselhuber NR, KIco JM, Verdoni AM, Lamprecht T, Sarkaria SM, et al. (2013) Notch signaling in acute promyelocytic leukemia. Leukemia.
8. Ye YZ, Zhang ZH, Fan XY, Xu XL, Chen ML, et al. (2013) Notch3 overexpression associates with poor prognosis in human non-small-cell lung cancer. Med Oncol 30: 595 